\title{
Lectures
}

Les comptes rendus

/

2012

\section{Jean Gadrey, Adieu à la croissance. Bien vivre dans un monde solidaire}

\author{
FRANÇOIS THOREAU
}

JEAN GADREY

\section{ADIEU}

À LA

CROISSANCE

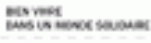

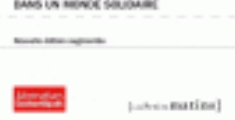

Jean Gadrey, Adieu à la croissance. Bien vivre dans un monde

solidaire, Paris, Les Petits Matins, 2011.

\section{Texte intégral}

\section{PDF}

Dans Adieu à la croissance, la proposition que fait Jean Gadrey consiste à faire l'inventaire à la fois d'un des problèmes les plus prégnants de notre époque, ainsi que des remèdes qui permettent ou permettraient d'y pallier. C'est une démarche intéressante par son ampleur et le vaste " aperçu hélicoptère » que Gadrey développe autour de la question de la croissance économique. Il ne s'agit pas de repartir dans des interrogations sans fin sur le bien-fondé, ou non, des politiques économiques de croissance quantitative, ni de tomber dans le « solutionnisme » béat, à grands coups de « y’a qu'à ». Au contraire, il s'agit d'établir un constat ferme et, surtout, des alternatives concrètes. Ainsi, le questionnement proposé est profondément actuel, comme en témoigne la préface de la nouvelle édition, où Gadrey réalise ce tour de force de formuler une série de questions très percutantes aux candidats à l'élection présidentielle, dont on serait excessivement curieux qu'un journaliste politique se décide à les leur poser sans fard, dans le blanc des yeux.

Tout au long de l'ouvrage, Gadrey déploie un exposé systématique et rigoureux, 
abondamment illustré, qui articule les limites du modèle actuel de croissance économique aux solutions qui devront nécessairement lui succéder. Dans une première partie (pp. 29-74), il aborde et récapitule les éléments, bien connus par ailleurs, qui conduisent au diagnostic général suivant, sans appel : la poursuite de la croissance est devenue un problème bien plus qu'une solution, et cause bien plus de problèmes qu'elle n'en résout. La seconde partie (pp. 77-124) est assez originale en ce sens que Gadrey y aborde et y documente la plus-value d'une société post-croissance, dans les termes actuels du débat public, c'est-à-dire essentiellement en matière de création d'emplois. Tenant à se démarquer de certains courants décroissants, Gardey soutient qu'il faut rendre des formes de décroissance désirable, et que cela passe par des emplois de qualité et une revalorisation de savoir-faire conviviaux et innovants, qui peuvent apporter la satisfaction et la reconnaissance du travail bien fait. Les gisements d'emplois sont considérables dans une bonne partie des secteurs d'activité économiques, dont Gadrey dresse un tableau exhaustif (pp. 115-117). Dans cette partie comme à d'autres endroits dans l'ouvrage, on retrouve cette idée centrale de sélectivité, salutaire pour le succès des idées " a-croissantes »; que doivent croître certains biens et services, pourvoyeurs de bien-être et d'épanouissement, parallèlement à la décroissance d'autres activités excessivement productivistes. Toute croissance ou décroissance n'est donc pas bonne à prendre dans l'absolu.

Il faut souligner, dans ces parties, l'attention portée aux termes et l'accompagnement du lecteur, qui se voit guidé dans les méandres des indicateurs que sont la « croissance économique » et le « produit intérieur brut », mécanos compliqués dont Gadrey démonte efficacement les rouages, de manière toujours très lisible pour le non-initié. Ainsi, par exemple, du calcul de la " productivité ». Qu'estce qu'un travailleur productif ? Quand un État est-il productif ? Ces questions reviennent sans cesse dans le débat public et font régulièrement l'objet de chiffrages divers. On reste stupéfait, à la lecture de l'encadré (pp. 88-89), qui explique l'amateurisme - c'est le mot qui vient spontanément en tête - avec lequel est calculée ou mesurée une notion si prégnante dans les querelles actuelles, sur fond de crise économique.

Les troisième et quatrième parties, plus ténues en volume (pp. 129-154 et 157-186) se permettent alors de reposer la question de la croissance dans un cadre plus large, plus utopiste sans doute, et à la fois dans des formulations qu'impose l'ampleur des crises actuelles. Là où, dans les écrits sur la décroissance, ce type d'interventions peut parfois sembler relever du fantasme ou, à tout le moins, entretenir une certaine " déconnexion » avec la réalité, Gadrey démontre son souci constant de coller aux réalités institutionnelles existantes, de localiser les alternatives possibles au travers des structures en place, de prendre acquis de ce qui, déjà, fonctionne, plutôt que de réinventer sans fin la grande roue de la machine à utopies.

Ce côté pragmatique, presque terre-à-terre, très marqué en tous cas par les réalités de terrain, est une constante de l'ouvrage de Gadrey, qui donne à voir l'état des critiques, des possibilités et des alternatives à la croissance économique. D'un côté, on pourrait lui en faire le reproche, l'ouvrage ne brillant pas nécessairement par ses qualités littéraires hors-norme ni par un style foudroyant. Ce serait toutefois, dans le cas d'espèce, un mauvais procès, tant l'exposé y perdrait sans doute en didactique et en clarté - ce qui sont sans doute ses principales vertus. Non content de proposer une somme théorique sur les vertus d'une politique « a-croissanciste », Gadrey fait de son ouvrage un véritable manuel, un incitant à l'action pratique et concrète, à destination de celui qui veut se familiariser avec les notions de croissance et de décroissance, peser le pour et le contre, et fonder une action de terrain sur un ensemble d'arguments concrets et solidement étayés. À commencer par la possibilité d'une telle action, d'un projet de société alternatif, que Gadrey s'emploie à démontrer avec soin ; " La convergence est possible, écrit-il, du local à l'international, pour sortir d'une crise systémique qui sera probablement longue, entre les mille réseaux de la société civile, 
les mille initiatives de l'ESS [économie sociale et solidaire], les valeurs de service public, de biens communs, de gratuité et de réciprocité, et des institutions et partis politiques ouverts à ces acteurs et à ces valeurs » (p. 180).

Ce faisant, Gadrey montre son attachement au « cri » émis par les manifestants de Seattle, sur lequel se sont appuyés Philippe Pignarre et Isabelle Stengers dans un essai très important ${ }^{1}$ : "Un autre monde est possible ! ». Gadrey s'attache ainsi à montrer la praticabilité de voies alternatives, n'hésitant pas à porter la controverse sur le terrain des économistes en usant de nombreux graphiques et statistiques ${ }^{2}$. En conclusion, dans un ouvrage à vocation très généraliste, Gadrey propose un tour complet d'une question brûlante d'actualité, à la lueur des crises multiples ; le problème de la croissance économique, auquel les alternatives sont porteuses de perspectives d'émancipation, qui devront articuler autant de réponses créatives et audacieuses que la poursuite infinie de la croissance dans un monde fini n'en pose. Il établit l'indispensable désirabilité des modèles alternatifs, que ce soit à cadres constants (création d'emplois), ou à cadres renouvelés (un nouveau projet de société). En ce sens, si l'ouvrage propose peu d'éléments fondamentalement novateurs, il réalise une très bonne synthèse et un incitant efficace à l'action militante de terrain.

\section{Notes}

1 Et dont il faut aujourd'hui hériter, selon la proposition de Pignarre et Stengers (in $L a$ sorcellerie capitaliste. Pratiques de désenvoûtement, Paris : Les empêcheurs de penser en rond, 2005, pp. 9-18).

2 Au-delà des limites heuristiques évidentes de telles ressources, elles permettent d'éviter de succomber à une dictature des chiffres et des courbes, dont abusent certains économistes orthodoxes et, de la sorte, de donner une prise aux argumentaires décroissants ou acroissants, lorsqu'il s'agit de croiser le fer avec ceux-ci.

\section{Pour citer cet article}

Référence électronique

François Thoreau, « Jean Gadrey, Adieu à la croissance. Bien vivre dans un monde solidaire », Lectures [En ligne], Les comptes rendus, 2012, mis en ligne le 04 mars 2012, consulté le 05 mars 2012. URL : http://lectures.revues.org/7731

\section{Rédacteur}

\section{François Thoreau}

Aspirant du F.R.S.-FNRS en sciences politiques et sociales, au centre de recherche Spiral, au sein du département de science politique de la Faculté de droit, à l'Université de Liège. Membre fondateur du réseau belge pour les Sciences \& Technologies en Société (STS)

Articles du même rédacteur

Augusto Forti, Aux origines de l'Occident : machines, bourgeoisie et capitalisme [Texte intégral]

Frédéric Gaillard, Pièces et main d'oeuvre, L'industrie de la contrainte [Texte intégral] Jocelyne Porcher, Vivre avec les animaux. Une utopie pour le XXIe siècle [Texte intégral]

Tous les textes

\section{Droits d'auteur}

(C) Tous droits réservés 